\title{
Integralidade e Interdisciplinaridade na Formação de Estudantes de Medicina
}

\author{
Comprehensive and Interdisciplinary \\ Medical Training
}

\author{
Renata Newman Leite Cardoso dos Santos ${ }^{I}$ \\ Kátia Suely Queiroz Silva Ribeiro ${ }^{I}$ \\ Ulisses Umbelino dos Anjos ${ }^{I}$ \\ Danyelle Nóbrega de Farias ${ }^{l}$ \\ Eleazar Marinho de Freitas Lucenal
}

\section{PALAVRAS-CHAVE}

- Educação Médica;

- Integralidade na Saúde;

- Comunicação Interdisciplinar.

\section{KEYWORDS}

- Medical Education;

- Comprehensive Health Care;

- Interdisciplinary

Communication

Recebido em: 16/09/2014

Aprovado em: 18/04/2015

\section{RESUMO}

Este artigo objetiva investigar de que forma os alunos de Medicina estão sendo formados quanto à integralidade e à interdisciplinaridade. O estudo teve abordagens qualitativa e quantitativa. Inicialmente, foi realizado um grupo focal com estudantes do último ano do curso de Medicina da Universidade Federal da Paraíba; posteriormente, todos os estudantes matriculados no último ano do curso responderam a um questionário elaborado pelos pesquisadores, com questões sobre o perfil sociodemográfico e estudantil, e sobre a integralidade e interdisciplinaridade na formação médica. As questões avaliaram conceito, prática, aptidão profissional e contribuição da extensão universitária para a integralidade e interdisciplinaridade, as quais se mostraram limitadas na formação dos estudantes. Os resultados apontam a necessidade de priorizar a interação entre teoria e prática; investir na formação dos docentes de modo a capacitá-los para atuação/ensino da integralidade e interdisciplinaridade; organizar as atividades para que possam garantir encontros em componentes curriculares obrigatórios, realizando atividades conjuntas numa perspectiva interdisciplinar; diversificar mais os cenários de prática, para que contemplem a integralidade no cuidado e a atuação interdisciplinar, em todos os níveis de atenção à saúde.

\begin{abstract}
This article aims to understand how medical students are being trained in terms of a comprehensive and interdisciplinary perspective. The study involved qualitative and quantitative approaches and began with a focus group with final-year medicine students from the Federal University of Paraíba. Subsequently, all students enrolled for the final year of the course completed a questionnaire prepared by the researchers with questions about their sociodemographic and student profile, and comprehensive and interdisciplinary medical training. The questions evaluated the concept, the practice, professional competence and the contribution of community outreach programs in relation to a comprehensive and interdisplinary training approach, which appears to be limited. The results indicate the need to: prioritize greater interaction between theory and practice; invest in teacher training to enable them to engage and teach comprehensive and interdisplinary health care; organize activities so that they can ensure meetings in the required curricular components, conducting joint activities in an interdisciplinary perspective; greater diversity of practice settings so as to include comprehensive care in an interdisciplinary context at all levels of health care.
\end{abstract}




\section{INTRODUÇÃO}

Os profissionais da área da saúde, em especial os médicos, na construção histórica de sua formação profissional, tiveram como cenários de prática em seus cursos de graduação ambientes hospitalares e clínicas, em que predominava um modelo de atenção individualizado, especializado, que fragmenta o indivíduo em sistemas. Esse modelo de formação mostrou-se inadequado para atuar nos problemas de saúde da coletividade e, mais recentemente, para atuar no Sistema Único de Saúde (SUS) ${ }^{1}$.

Com o SUS, o modelo de saúde vigente passou a ser também, pelo menos teoricamente, o modelo de formação profissional. Profissionais capazes de atuar integralmente no cuidado à saúde do indivíduo e da comunidade passaram a ser um dos requisitos para a atuação em saúde ${ }^{2}$. A integralidade pode ser compreendida como um termo polissêmico, carregando em si sentidos e representações verbalizados e sentidos por usuários, associados ao compromisso dos profissionais de saúde em suas práticas ${ }^{3}$. Dentre estes sentidos, destacam-se: visão integral do ser humano; ações de prevenção, promoção, recuperação e reabilitação da saúde; e articulação das ações de saúde nos três níveis de assistência ${ }^{4}$.

A integralidade nega a fragmentação frente aos problemas de saúde, na qual cada profissional se responsabiliza por uma parte do indivíduo. Para concretizar a integralidade na saúde, necessita-se de ações interdisciplinares. A interdisciplinaridade foi abordada por vários autores ${ }^{5,6,7}$, sendo comum entre eles a concepção de troca de saberes entre os conhecimentos de duas ou mais disciplinas. Oliveira et al..$^{8}$ referem-se à interdisciplinaridade como "uma troca intensa de saberes profissionais especializados em diversos campos, exercendo [...] uma ação de reciprocidade e mutualidade, [...] ou seja, é a substituição de uma concepção fragmentada e distante por uma visão unitária e abrangente sobre o ser humano"(p.29).

Considerando que os aspectos da interdisciplinaridade são necessários não apenas na formação em saúde, mas, sobretudo, na prática profissional, observa-se que esta atuação precisa ser trabalhada para capacitar os futuros profissionais, tornando-os aptos a respeitar a disciplina do outro; colocar-se no lugar do outro; aceitar sugestões; respeitar as limitações dos demais profissionais; respeitar as competências do outro; comprometer-se com o SUS; ouvir a opinião do outro; pensar criticamente; ser humilde; estar pronto para aprender e mudar, se necessário; respeitar as diferenças; trabalhar com ética e atuar com liderança pactuada e democrática ${ }^{9}$.

Diante do modelo atual de saúde, no qual a Estratégia Saúde da Família (ESF) representa a principal porta de entrada dos usuários no sistema e o modelo que reorienta a atenção à saúde no Brasil, os profissionais que nela atuam devem estar aptos a atuar de forma integral e em equipes interdisciplinares, contribuindo para a prestação de um cuidado em saúde mais humanizado ${ }^{10}$. Assim sendo, o objetivo deste artigo é analisar a formação de estudantes do curso de Medicina de uma Instituição de Ensino Superior no que diz respeito à integralidade e à interdisciplinaridade.

\section{METODOLOGIA}

Este artigo é um recorte do trabalho de dissertação intitulado: "A Integralidade e a Interdisciplinaridade na Formação dos Profissionais da Estratégia Saúde da Família", estudo transversal, exploratório, descritivo e inferencial, com abordagens qualitativa e quantitativa. $\mathrm{O}$ recorte utilizado tem como enfoque a formação dos profissionais médicos. A pesquisa foi feita em duas etapas: no primeiro momento, foi realizada a técnica do grupo focal (GF), que consiste na interação entre os participantes e o pesquisador, objetivando colher dados com base na discussão focada em tópicos específicos e diretivos, problematizando o tema estudado ${ }^{11}$. Em ambos os momentos, buscava-se conhecer a percepção dos discentes quanto à formação prática e teórica para atuação profissional relacionada à integralidade e à interdisciplinaridade.

Participaram do GF dez estudantes do último ano do curso de Medicina da Universidade Federal da Paraíba. Eles foram selecionados de forma aleatória, foram contatados previamente e, após a explicação dos objetivos do estudo, foram convidados a participar da pesquisa. Posteriormente, foi agendado horário e dia compatível para a participação de todos.

Os dados do grupo focal foram coletados por meio de um gravador, que registrou as discussões acerca do tema proposto. Depois, as gravações foram transcritas integralmente para viabilizar a análise dos dados ${ }^{12}$. Após as explicações, os participantes assinaram o Termo de Consentimento Livre e Esclarecido, atestando sua concordância em participar do estudo.

O material coletado foi analisado pela técnica do Discurso do Sujeito Coletivo ${ }^{13}$, extraindo-se de cada um dos depoimentos as Ideias Centrais (IC) e suas correspondentes Expressões-Chave (ECH). Com as ECH e IC semelhantes foram criados os discursos-síntese, redigidos na primeira pessoa do singular ou plural, representando a fala de um eu coletivo.

Na segunda etapa da pesquisa, foi utilizado um questionário, desenvolvido pelos autores, dividido em três partes: a primeira continha questões sobre o perfil socioeconômico (idade, sexo, estado civil, religião, cor/raça, renda familiar) e perfil estudantil (período do curso, participação em projeto de extensão). A segunda parte constou de um Questionário de Avaliação da Integralidade, composto por 16 assertivas sobre 
a formação para a integralidade, divididas em quatro dimensões: Base Conceitual, Preparo Prático, Aptidão Profissional e Contribuição da Extensão. Nas afirmativas sobre Base Conceitual, Aptidão Profissional e Contribuição da Extensão, os estudantes assinalaram o grau de concordância, variando de 0 - discordo totalmente a 10 - concordo totalmente; já nas afirmativas sobre Formação Prática, os estudantes assinalaram a frequência com que praticaram cada afirmativa, de 0 - nunca a 10 - sempre. A terceira parte, correspondendo ao Questionário de Avaliação da Interdisciplinaridade, foi composta por 14 afirmativas sobre a formação para a interdisciplinaridade, dividida em quatro dimensões, desenvolvidas de forma semelhante à segunda parte do questionário.

O valor obtido em cada dimensão foi calculado pelo escore médio, somando-se o valor assinalado em cada assertiva da dimensão e dividindo-se pelo número de assertivas da referida dimensão; desta forma, o valor obtido em cada dimensão pode variar de 0 a 10. Os escores obtidos em cada dimensão, como também o escore obtido no total de cada questionário, são classificados em três intervalos. Se o valor obtido estiver no intervalo $[0,00 ; 3,33]$, isto indica que esta dimensão tem baixa, ruim, pouca ou fraca contribuição para a formação para a integralidade ou interdisciplinaridade; se o valor obtido estiver no intervalo $[3,33 ; 6,66]$, a dimensão tem contribuição média ou mediana para a formação da integralidade ou interdisciplinaridade; por último, se o valor obtido estiver no intervalo $[6,66 ; 10,00]$, isto indica que a dimensão tem alta, boa, muita ou forte contribuição em formar para a integralidade ou interdisciplinaridade.

Participaram desta segunda etapa todos os estudantes que estavam matriculados no último ano do curso de Medicina no momento da coleta de dados, sendo excluídos: estudantes transferidos de outras instituições de ensino superior, aqueles que tivessem concluído outra graduação ou curso de nível técnico em saúde e os estudantes que já tivessem concluído suas atividades acadêmicas no período de coleta dos dados.

Para análise quantitativa, os dados coletados por meio do Questionário de Pesquisa foram inicialmente tabulados numa planilha eletrônica (Microsoft Office Excel 2007) e posteriormente transferidos para um pacote estatístico (software $R$, versão i386 2.15.1). Realizou-se a análise descritiva a partir de frequências absolutas e relativas para as variáveis do perfil estudantil e média \pm desvio padrão da média dos escores obtidos em cada dimensão do questionário, como também das médias obtidas no questionário de interdisciplinaridade como um todo.

O estudo foi submetido à Plataforma Brasil e posteriormente aprovado pelo Comitê de Ética em Pesquisa do Centro de Ciências da Saúde da Universidade Federal da Paraíba, sob o $\mathrm{n}^{\mathrm{o}}$ 0224/13. Todos os participantes do estudo assinaram o Termo de Consentimento Livre e Esclarecido, autorizando a realização e a publicação do estudo, sendo respeitada a autonomia e a garantia do anonimato dos participantes, assegurando sua privacidade, como regem as Resoluções 196/96 e 466/12 do Conselho Nacional de Saúde.

\section{RESULTADOS E DISCUSSÃO}

\section{Análise descritiva da amostra}

A amostra foi composta por 61 estudantes do curso de Medicina. A idade da amostra variou de 21 a 36 anos, com média de 24,6 $\pm 1,5$ anos. Quanto à variável sexo, verificou-se predominância do sexo masculino $(63,9 \%)$ em relação ao feminino $(36,1 \%)$, como apresentado na Tabela 1 . Esses resultados refletem a realidade encontrada no Enade 2004, em que, no curso de Medicina, observa-se prevalência do sexo masculino. A análise histórica de 1991-2004 ${ }^{14}$, apesar de evidenciar prevalência do sexo masculino nos cursos de Medicina no Brasil, mostrou uma tendência ao aumento de estudantes do sexo feminino nesses cursos.

TABELA 1

\begin{tabular}{|c|c|}
\hline $\begin{array}{r}\mathrm{T} \\
\text { Perfil sociodemográfico } \\
\text { Medicina. }\end{array}$ & liscentes de \\
\hline Variável & $\begin{array}{c}\text { Estudantes de } \\
\text { Medicina }\end{array}$ \\
\hline SEXO & $\%$ \\
\hline Masculino & 63,9 \\
\hline Feminino & 36,1 \\
\hline \multicolumn{2}{|l|}{ ESTADO CIVIL } \\
\hline Solteiro & 90,2 \\
\hline Casado & 4,9 \\
\hline Divorciado & 1,6 \\
\hline Viúvo & - \\
\hline Outro & 3,3 \\
\hline \multicolumn{2}{|l|}{ RELIGIÃO } \\
\hline Católico & 62,3 \\
\hline Evangélico & 18,0 \\
\hline Espírita & 3,3 \\
\hline Sem religião & 6,6 \\
\hline Ateu & 8,2 \\
\hline Outra & 1,6 \\
\hline \multicolumn{2}{|l|}{ COR/RAÇA } \\
\hline Branca & 60,7 \\
\hline Preta & 3,3 \\
\hline Amarela & 4,9 \\
\hline Parda & 31,1 \\
\hline Indígena & - \\
\hline \multicolumn{2}{|c|}{ RENDA FAMILIAR (em salários mínimos) } \\
\hline$<1 \mathrm{SM}$ & 1,6 \\
\hline $1-2 \mathrm{SM}$ & 1,6 \\
\hline
\end{tabular}




$\begin{array}{lr}3 \text { - } 4 \text { SM } & 4,9 \\ \geq 5 \text { SM } & 91,8 \\ \text { TEMPO DE GRADUAÇÃO } & \\ \text { Previsto } & 98,4 \\ \text { Maior que o previsto } & 1,6 \\ \text { PARTICIPA DE PROJETO DE EXTENSÃO } & 86,9 \\ \text { Sim } & 13,1\end{array}$

Fonte: Dados da pesquisa. João Pessoa (PB), 2013.

Quanto ao estado civil, a maioria da amostra declarou ser solteira (90,2\%). Em relação à religião, a prevalente foi a católica $(62,3 \%)$, seguida pela evangélica $(18,0 \%)$. Na variável cor / raça, a maioria dos estudantes declarou ser branca, com $60,7 \%$, seguida pelos que declararam cor/raça parda, com 31,0\%. No tocante à renda familiar, a maioria da amostra do estudo $(91,8 \%)$ afirmou ter renda de cinco salários mínimos ou mais.

O tempo de graduação estava de acordo com o tempo previsto para o curso em $98,4 \%$ dos estudantes que participaram do estudo. Quanto à participação em projetos de extensão, $86,9 \%$ da amostra afirmou participar ou ter participado de algum projeto.

\section{Concepções e práticas integrais}

Este item apresenta as concepções e os cenários de prática dos estudantes de Medicina quanto à integralidade, por meio dos seus discursos e dos escores obtidos nas dimensões Base Conceitual e Preparo Prático do Questionário de Integralidade.

\section{Conceito de integralidade}

Quando questionados sobre o que entendem por integralidade na atenção à saúde, os estudantes expressaram três Ideias Centrais (IC) em suas falas: "atuação multiprofissional" (IC); "visão integral do ser humano" (IC); e "atenção nos três níveis da assistência" (IC). Cada IC apresentada deu origem a um Discurso do Sujeito Coletivo (DSC), como pode ser observado a seguir:

"É a interação de toda uma equipe, é o trabalho das especialidades que são da área da saúde, basicamente é isso" (DSC)

"É dar atenção ao paciente como um todo, biopsicossocial, observando o contexto onde ele está inserido" (DCS)

"Considero como um aspecto amplo, o acompanhamento longitudinal da comunidade, do paciente; é desviar o foco da atenção biomédica, prezando a profilaxia, a prevenção e a promoção de saúde, a reabilitação e a redução de danos à saúde" (DSC)
Nos discursos dos discentes, pode-se observar o conceito apreendido por eles sobre a integralidade na atenção à saúde de forma ampliada, negando o reducionismo, como também o foco em ações de assistência à saúde, apontando a necessidade de trabalhar com outros profissionais como meio de atingir a integralidade em saúde. Reafirmando o que os discursos nos revelam, os escores desses estudantes na dimensão Base Conceitual demonstram que a formação teórica para a integralidade se apresenta boa $(8,75 \pm 1,36)$. Os resultados apontam que a formação dos estudantes de Medicina favorece um conceito próximo do que se espera de um profissional que atue na atenção à saúde com foco na integralidade.

\section{A prática da integralidade}

A prática da integralidade foi vivenciada de formas diferentes pelos estudantes de Medicina participantes do grupo focal. Duas IC contrárias foram formadas quando se questionou: "Em qual (quais) momento(s) a integralidade foi vivenciada na sua formação acadêmica?" IC - "Nas disciplinas teóricas do primeiro período e nos estágios da Atenção Básica"; IC - "Nos estágios da atenção secundária e terciária". Os DSC seguintes revelam que as práticas para a integralidade se baseiam em ações pontuais e não representam uma formação que tenha como foco a integralidade na atenção à saúde de forma transversal em todo o percurso acadêmico, sendo impossível desenvolvê-la em todos os espaços e cenários de ensino-aprendizagem:

\footnotetext{
"Desde o começo da nossa formação, no campo da saúde coletiva, em estágios no PSF, nas visitas familiares, como também nas aulas teóricas do primeiro período... Já no hospital você não aplica esses conceitos não" (DSC)

\begin{abstract}
"No curso de Medicina a gente vê mais isso no hospital, na parte das enfermarias... essa visão de integralidade foi mais intensa nas atenções secundária e terciária, apesar de o ideal ser começar na primária" (DSC)
\end{abstract}

Os discursos mostram vivências diferentes dos participantes quanto à integralidade: para alguns, ela foi vivenciada na atenção básica e negligenciada na atenção secundária e terciária; para outros discentes, a integralidade foi praticada nos níveis secundário e terciário, em detrimento da atenção básica. Este fato remete à ideia de que, neste curso de graduação, a formação não tem como foco a integralidade na atenção à saúde do usuário do sistema, ficando as práticas integrais a cargo de docentes que têm esta visão de atenção à saúde. Desta forma, os alunos que têm contato com tais profissionais conseguem assimilar e vivenciar a integralidade. 
Outro destaque nos discursos apresentados é o fato de que alguns estudantes avaliam que a atenção terciária tem maior capacidade de praticar a integralidade, em detrimento da atenção básica. Nogueira e Guedes ${ }^{15}$ compreendem que a atenção hospitalar é um cenário que privilegia o olhar sobre o corpo, fragmentando-o em especialidades, desconsiderando os aspectos psíquicos, culturais e sociais dos sujeitos. Considerando-se que o olhar integral sobre o sujeito hospitalizado se torna muito mais difícil, em face do distanciamento da sua realidade de vida, que as ações nesses serviços têm um enfoque extremamente curativo e que a interação com os demais níveis de atenção do sistema é precária, questiona-se a afirmação desses estudantes em relação a terem vivenciado a integralidade no ambiente hospitalar mais do que na atenção básica. Isto suscita, ainda, o questionamento acerca da concepção de integralidade que norteia tal afirmação.

Na dimensão Preparo Prático do questionário, os estudantes classificam sua formação prática como boa $(7,31 \pm 1,50)$ para a integralidade na atenção à saúde. Como mencionado acima, para os estudantes que participaram do grupo focal, a prática integral ora estava nas ações hospitalares, ora estava atrelada às práticas na atenção básica; entretanto, essa prática não é visualizada pelos mesmos estudantes nos diferentes cenários de práticas com as quais eles têm contato.

Costa et $a l .{ }^{16}$ avaliam que as escolas médicas que não têm como princípio, em seus projetos político-pedagógicos, método ou estratégia de ensino, o campo do cuidado em saúde e a centralidade do usuário junto ao seu processo avaliativo são vistas como escolas que têm baixo poder de alterar a hegemonia da atual organização do trabalho educacional em saúde e do modelo de saúde tradicional.

Espera-se que as escolas de formação médica - e das demais graduações em saúde - tenham currículos que objetivem superar os modelos tradicionais de formação, que integrem as unidades produtoras de cuidado e seus contextos socioculturais, possibilitando o envolvimento com a realidade onde o serviço está inserindo, tornando-se escolas com formação mais integral. No curso de Medicina avaliado, o currículo é integrado, mas ainda são evidentes lacunas na formação quanto à integralidade.

\section{Aptidão profissional para a integralidade}

A Aptidão Profissional foi investigada por meio do Questionário para a Integralidade. Nesta dimensão, os estudantes avaliaram seu preparo para atuar integralmente na atenção à saúde quando forem profissionais como bom $(8,37 \pm 1,55)$, indicando que se sentem aptos.
De acordo com esse resultado, pode-se deduzir que a formação desses estudantes possibilitou que se sintam capacitados para exercer suas práticas profissionais integralmente na atenção à saúde. Entretanto, o discurso dos estudantes quanto à sua prática na integralidade demonstra fragilidades para uma atuação integral no que tange tanto às concepções que a norteiam, quanto à vivência que a formação lhes proporcionou. Este fato remete a dificuldades que esses futuros profissionais podem ter ao exercerem a profissão na perspectiva do cuidado integral, uma vez que na prática estudantil eles não conseguem vivenciar plenamente a integralidade.

\section{Contribuição dos projetos de extensão}

No questionário para a Integralidade, a última dimensão avaliada foi a Contribuição da Extensão. Por extensão universitária entende-se: "[...] processo educativo, cultural e científico que articula o ensino e a pesquisa de forma indissociável e viabiliza a relação transformadora entre universidade e sociedade" $(p .5)^{17}$.

Nesta dimensão, os estudantes de Medicina $(5,30 \pm 3,54)$ classificaram como média a contribuição da extensão universitária para sua formação na integralidade. A extensão universitária representa uma das ações do processo formativo acadêmico, que, através de vivências, promove trocas e relações sociais nos cenários próximos à realidade dos sujeitos. É um espaço de reflexão crítica que faz repensar ações acadêmicas frente às demandas sociais e à formação de profissionais ${ }^{18}$. As atividades de extensão universitária podem tanto refletir o modelo assistencialista hegemônico nos serviços de saúde, como favorecer junto aos estudantes práticas de saúde que contemplem a formação de um profissional crítico, reflexivo, problematizador, comprometido com ações de saúde mais integrais.

De acordo com a classificação que os estudantes de Medicina atribuíram à contribuição das atividades de extensão universitária, estas, possivelmente, estão vinculadas a ações de saúde assistencialistas, que pouco contribuem para a formação de profissionais humanizados, comprometidos com a realidade social e com a integralidade nas ações de saúde. Dessa forma, a extensão universitária com caráter assistencialista passa a ser um obstáculo para a efetividade de uma formação mais voltada à integralidade da saúde dos sujeitos.

\section{Barreiras para a integralidade}

O discurso dos sujeitos do curso de Medicina permitiu conhecer as principais barreiras identificadas por eles para vivenciar a integralidade na formação. Para esses discentes, as "dificuldades de trabalho no SUS" representam uma barreira para a formação na integralidade, presente no seguinte DSC: 
"Não há incentivo para o profissional ser integral, profissionais do Sistema Único de Saúde, eles têm uma função que não é integral, o mercado de trabalho e o próprio SUS não incentivam... A informação não vem desde a atenção básica; não existe uma integração da atenção primária, da atenção secundária, é tudo separado... se colocar um médico da atenção secundária ou da atenção terciária pra entender o contexto social, ele não vai entender... é a referência e a contrarreferência, o que na prática não é feito" (DSC)

Como mostrado acima, não existe articulação entre os níveis de atenção à saúde, distanciando as práticas de saúde da integralidade. Em estudo realizado com profissionais que trabalham na ESF, Machado et al. ${ }^{19}$ evidenciaram que o sistema de referência e contrarreferência consiste numa dificuldade no trabalho no SUS, tendo em vista que ele não existe efetivamente nos serviços de saúde. Os autores afirmam que a estrutura dos serviços proporciona uma demora na referência e a não visualização da contrarreferência. Desta forma, a lacuna existente na comunicação nos serviços de saúde compromete a resolutividade das ações de saúde.

Ainda sobre o discurso anterior, observa-se que, para os discentes, os serviços de saúde, na prática, não contribuem para uma prática integral. Pires et al. ${ }^{3}$, analisando a atenção à saúde integral, destacaram que os gestores em saúde no âmbito municipal não conseguem efetivar as mudanças necessárias para uma prática integral, visualizando o cuidado em saúde numa perspectiva apenas biológica, o que representa um obstáculo no processo de trabalho na ESF para o atendimento integral ao usuário.

$\mathrm{Na}$ segunda IC formada pelos estudantes, observa-se como barreira para a integralidade: "empecilhos na formação profissional e formação dos docentes na visão biomédica", ilustrada no DSC a seguir.

\footnotetext{
"Falta uma integração das disciplinas, o próprio hospital universitário não permite, até dentro do próprio setor não existe integralidade. Tem uma demanda muito grande de pacientes, falta tempo... A formação dos nossos professores, a maioria tem uma formação numa visão antiga, sem essa visão integral" (DSC)
}

O discurso desses estudantes evidencia que a organização da formação, com disciplinas que não se integram, dificulta a formação para a integralidade, e a vivência no hospital universitário sem a perspectiva da integralidade contribui para a permanência do modelo hegemônico de saúde, com uma visão fragmentada do sujeito. A grande demanda de pacientes e a falta de tempo para um atendimento mais humanizado e integral também dificultam a formação dos profissionais nos moldes da integralidade na saúde.

A formação dos docentes que atuam nas escolas médicas, em geral, foi desenvolvida no modelo tradicional de saúde, que prioriza as práticas de saúde centradas em procedimentos, desvalorizando o usuário como protagonista do seu processo saúde-doença. Assim, a formação dos docentes apresenta-se como uma barreira para a integralidade. Entretanto, também se observam como empecilhos no trabalho docente: despreparo pedagógico do docente; organizações tradicionais de ensino que priorizam a organização disciplinar e o ensino que tem como protagonista o professor, acarretando avaliação punitiva e opressora; vínculo precário das contratações parciais; resistência dos docentes às mudanças; complexidade dos cenários de aprendizagem; insegurança e corporativismo entre os docentes médicos ${ }^{20}$.

\section{Concepções e práticas interdisciplinares}

Este item apresenta as concepções e os cenários de prática dos discentes por intermédio dos seus discursos e dos escores obtidos nas dimensões Base Conceitual e Preparo Prático do Questionário de Interdisciplinaridade.

\section{Conceito de interdisciplinaridade}

Os estudantes de Medicina, quando questionados sobre o que entendem por interdisciplinaridade, revelaram uma IC: "trabalho em equipe, troca de conhecimentos e reconhecer os limites de cada um". Esse conceito se aproxima das concepções de Japiassú ${ }^{5}$, Batista ${ }^{6}$ e Zabala ${ }^{7}$ no que se refere à troca de conhecimentos entre integrantes de uma mesma equipe, e das concepções de Suape ${ }^{9}$ no tocante a reconhecer os limites de cada profissional, indicando que os estudantes apreenderam o conceito de interdisciplinaridade.

Garcia et al. ${ }^{21}$, em estudo com alunos de Medicina, identificaram em seus discursos concepções semelhantes sobre a interdisciplinaridade, conceituando-a como a união entre as diversas áreas do conhecimento para atingir o mesmo objetivo. Essa IC deu origem ao seguinte DSC:

"É integrar várias especialidades de determinada profissão ou mais profissões, é o trabalho conjunto dos profissionais de saúde, médico, enfermeiro, fisioterapeuta, psicólogo, ou seja, o trabalho em equipe; mas que exista certo espaço definindo o trabalho de cada um; tem que ter uma troca de conhecimentos, que intervenha um no outro, mas que cada um saiba seu papel; que todos tenham a mesma finalidade em prol do paciente" (DSC) 
Os estudantes que participaram do grupo focal parecem ter uma concepção de interdisciplinaridade coerente com o proposto pelos estudiosos do tema. Entretanto, na avaliação do Questionário de Interdisciplinaridade, na dimensão Base Conceitual, a compreensão que eles apresentaram sobre a interdisciplinaridade foi mediana $(5,04 \pm 0,95)$. Na maioria dos casos, nesta avaliação, os estudantes compreendiam a interdisciplinaridade como a prática conjunta de profissionais de especialidades diferentes, porém não consideravam o diálogo e a troca de conhecimentos como essenciais à concretização de práticas interdisciplinares. Desta forma, para os estudantes que responderam ao questionário, a concepção de interdisciplinaridade é mais condizente com a prática da multidisciplinaridade, facilmente observada nos serviços de saúde, mas que não consegue efetivar a atuação interdisciplinar.

\section{A prática e a aptidão para a interdisciplinaridade}

Percebe-se uma dicotomia no discurso dos estudantes: alguns destacam espaços de prática interdisciplinar, enquanto outros consideram nunca terem vivenciado a interdisciplinaridade. Para os primeiros, a prática interdisciplinar está presente nos seguintes cenários: "atenção básica, secundária e terciária, projetos de extensão, PET-Saúde", dando origem ao DSC que segue:

\footnotetext{
“Tanto na atenção básica, como na atenção secundária e terciária, vê-se muito isso... no rodízio de clínica médica, em todos os espaços a gente teve contato com a interdisciplinaridade, nos projetos de extensão, no PET-Saúde" (DSC)
}

Apesar de evidenciarem locais de prática interdisciplinar, os estudantes classificaram a prática para a interdisciplinaridade como média $(5,33 \pm 2,20)$. Ao analisar o discurso acima, é importante mencionar que a concepção de interdisciplinaridade desenvolvida na clínica médica por esses alunos diz respeito, na realidade, à atuação multiprofissional, onde vários profissionais olham o paciente de modo fragmentado, de acordo com sua especialidade. Nesse contexto, as trocas de informações sobre o paciente, na maioria das vezes, são feitas com base em pareceres, contrariando o que se espera de uma ação interdisciplinar.

Outro aspecto importante é que os discentes relatam "ter contato" com a interdisciplinaridade. O contato apenas não é capaz de garantir uma prática interdisciplinar, uma vez que remete à ideia de encontros esporádicos ou poucas oportunidades de atuar interdisciplinarmente. A prática interdisciplinar deve ser construída dia a dia na vivência do estudante, de modo que se torne uma atitude constante no seu cotidiano profissional e não uma atitude esporádica.
Mesmo diante desta fragilidade na prática interdisciplinar, observa-se que os projetos de extensão e o PET-Saúde são importantes cenários que favorecem o contato com a interdisciplinaridade. Estes espaços mostram-se verdadeiros oásis num contexto de formação profissional onde os estágios curriculares pouco permitem/favorecem a interdisciplinaridade.

A aquisição do conhecimento com pessoas de diferentes áreas parece ser uma experiência que abrange a compreensão do trabalho coletivo e da complexidade da realidade que se apresenta nos cenários de saúde ${ }^{22}$. A formação profissional interdisciplinar é de extrema relevância, tendo em vista que a identidade profissional vai se fortalecendo à medida que os estudantes são expostos a situações comuns de aprendizagem. A complexidade da prática compartilhada por estudantes de diferentes áreas possibilita, frente a um mesmo problema, olhares diferentes, que ora se complementam, ora se confrontam, mas proporcionam um olhar mais ampliado de compreensão da realidade na saúde ${ }^{23}$.

A segunda IC refere-se a: "não vivenciei a interdisciplinaridade na formação", evidenciada pelo seguinte DSC:

\begin{abstract}
"Esse conceito de troca de conhecimentos eu não vi muito não, eu vi mais assim, um profissional trabalhando sozinho e uma vez ou outra vem outro profissional; mas assim com troca de conhecimentos eu não vi... No hospital a gente vê bastante isso, pela forma dos pareceres que se pede, só com parecer, com discussão não, não tem assim um grupo de pessoas conversando... a gente também não viu no PSF a interdisciplinaridade" (DSC)
\end{abstract}

Na terceira IC, observa-se que "interdisciplinaridade é abordada apenas teoricamente". O DSC formado foi o seguinte: "A gente teve muita aula no sentido de dizer à gente que era
importante a interdisciplinaridade, mas a gente não teve aula
de como usar isso..." (DSC)

Diante das IC formadas, observa-se grande fragilidade na formação para a interdisciplinaridade. As ações desenvolvidas são atividades pontuais de alguns docentes, mas que não estão presentes na formação de todos os alunos de Medicina dessa instituição de ensino. Assim, a visão interdisciplinar só é apreendida pelos estudantes que têm contato com docentes que propiciam a interdisciplinaridade ou em projetos de extensão e/ou PET-Saúde. Evidencia-se, assim, que o curso não tem uma formação voltada para a interdisciplinaridade, o que vai de encontro ao que se espera de um profissional que atuará no sistema de saúde brasileiro. 
Fazenda ${ }^{24}$ assinala que interdisciplinaridade não se ensina nem se aprende, simplesmente vive-se, exerce-se e por isso exige uma nova pedagogia, o que implica o envolvimento de todos os docentes na construção de estratégias de ensino que possibilitem a vivência interdisciplinar.

\section{Aptidão profissional}

A dimensão Aptidão Profissional foi avaliada por meio do questionário e classificada como média para os estudantes de Medicina $(4,42 \pm 1,37)$. A capacidade de atuar após a formatura está fortemente atrelada ao preparo prático que se tem enquanto graduando. Desta forma, a prática limitada para a interdisciplinaridade acarreta uma percepção e uma possibilidade de atuação interdisciplinar na vida profissional também limitadas.

\section{Contribuição dos projetos de extensão}

Os projetos de extensão universitária foram classificados como médios em contribuir para a formação interdisciplinar $(6,22 \pm 4,00)$. Como mencionado, as ações de extensão universitária possibilitam a prática das ações de saúde em ambientes próximos da realidade dos sujeitos participantes, favorecendo a aproximação dos discentes com a realidade de saúde da população. A extensão universitária contribui para a formação interdisciplinar quando esta possibilita a atuação conjunta de alunos de diversos cursos de graduação, trabalhando de forma dialógica e compartilhando objetivos comuns. Desta forma, os projetos de extensão que propiciam a prática da interdisciplinaridade garantem uma formação diferenciada aos alunos, uma vez que, nos cenários de prática disponibilizados pelo currículo mínimo dos cursos estudados, a prática interdisciplinar não é contemplada em sua totalidade.

\section{Barreiras para a interdisciplinaridade}

Os acadêmicos identificaram como barreiras para vivenciar a interdisciplinaridade na formação: "entraves do SUS", "falta de diálogo" e "falta de iniciativa do médico (liderança)". A primeira barreira para a integralidade gerou o seguinte DSC:

\section{“O modo como o serviço é montado, a não funcionalidade do sistema de saúde, não tem um caminho que possa encaminhar o paciente para o serviço" (DSC)}

Neste discurso, os estudantes reduzem a interdisciplinaridade ao encaminhamento de pacientes para outros níveis de assistência ou outros profissionais da rede de serviços, distanciando-se do conceito de interdisciplinaridade.

A segunda IC revela como barreira para a interdisciplinaridade: "falta de diálogo", conforme expressa o seguinte DSC:
"A gente acaba que não tem essa conversa e a gente não sabe o que cada um está fazendo, então eu acho que essa é a principal barreira, a falta de conversa..." (DSC)

Uma das premissas para a interdisciplinaridade ocorrer é o diálogo. Segundo Freire ${ }^{25}$, só é possível haver diálogo se houver um pensar crítico, esperança e humildade, pois a autossuficiência é incompatível com o diálogo. Sem diálogo não há troca de conhecimentos e não se consegue ouvir a opinião do outro, habilidades tão necessárias quando se trabalha com a saúde humana.

A terceira IC - "falta de iniciativa do médico (liderança)" - revela que, para os estudantes de Medicina, o médico é a liderança da equipe de saúde, e precisa partir dele a interdisciplinaridade, como identificado no seguinte DSC:

"Falta a iniciativa do médico como um líder na equipe" (DSC)

Alguns estudantes veem no médico uma liderança nas equipes de saúde. A liderança pactuada e democrática faz parte do agir interdisciplinar 9 , mas não é exclusividade médica; todos os profissionais de saúde precisam pactuar esta liderança, para que realmente haja ações interdisciplinares. Este discurso remete à questão do poder médico, visto como superior ao dos outros profissionais da equipe de saúde. No entanto, essa concepção distorce o termo interdisciplinaridade, que nega a superioridade de um profissional em relação a outro.

\section{CONSIDERAÇÕES FINAIS}

Os posicionamentos dos estudantes quanto à sua formação para a integralidade e interdisciplinaridade indicam lacunas na formação em Medicina, principalmente no que se refere a atividades práticas que contemplem atenção integral e interdisciplinar. De forma geral, a formação para a interdisciplinaridade apresenta-se mais deficitária que a formação integral, sendo raras as atividades que abrangem a atuação interdisciplinar dos estudantes. É preciso destacar, contudo, que as lacunas na atuação interdisciplinar comprometem o desenvolvimento de uma atenção integral à saúde, considerando-se a necessidade imperativa da interdisciplinaridade na busca da integralidade.

Fazendo uma analogia entre os resultados obtidos nos questionários e no grupo focal em relação aos conceitos de integralidade e interdisciplinaridade, verificou-se a dificuldade dos estudantes em elaborar um discurso acerca desses conceitos no grupo. É importante ressaltar que a elaboração de um discurso representa uma atividade complexa, em que é 
necessário ter conhecimento teórico sobre o tema e uma aproximação prática, a fim de melhorar a compreensão e possibilitar essa elaboração. Assim sendo, a dificuldade em elaborar os discursos indica limitações na formação, tanto teórica como prática, para a integralidade e interdisciplinaridade.

Os resultados apontam a necessidade de mudanças nos seguintes aspectos: priorizar a interação entre teoria e prática; investir na formação dos docentes de modo a capacitá-los para atuação/ensino da integralidade e interdisciplinaridade; organizar as atividades para que possam garantir encontros em componentes curriculares obrigatórios, realizando atividades conjuntas numa perspectiva interdisciplinar; diversificar mais os cenários de prática, para que contemplem a integralidade no cuidado e a atuação interdisciplinar, em todos os níveis de atenção à saúde.

Aponta-se como limitação do presente estudo não ter sido investigada a visão dos docentes e dos coordenadores do curso de Medicina quanto à formação para a integralidade e interdisciplinaridade. É necessário realizar mais pesquisas sobre o tema a fim de abordar este aspecto, como também é preciso expandir a análise para a formação dos demais profissionais da saúde.

\section{REFERÊNCIAS}

1. Almeida MM, Morais RP, Guimarães DF, Machado MFAS, Diniz RCM, Nuto SAS. Da Teoria à Prática da Interdisciplinaridade: a Experiência do Pró-Saúde Unifor e Seus Nove Cursos de Graduação. RBEM. 2012; 36(1 Supl.1):119-126.

2. Pinheiro R, Mattos RA. Os Sentidos da Integralidade na atenção e no cuidado à saúde. Rio de Janeiro: ABRASCO; 2001.

3. Pires VMMM, Rodrigues VP, Nascimento MAA. Sentidos da integralidade do cuidado na saúde da família. Rev. enferm. UERJ. 2010;18(4):622-7.

4. Silva RVGO, Ramos FRS. Integralidade em saúde: revisão de literatura. Ciência, Cuidado e Saúde. 2010;9(3):593-601.

5. Japiassu H. Interdisciplinaridade e patologia do saber. Rio de Janeiro: Imago; 1976.

6. Batista SHS. A interdisciplinaridade no ensino médico. Revista Brasileira de Educação Médica. 2006; 30(1):39-46.

7. Zabala A. Enfoque globalizador e pensamento complexo. Uma proposta para o currículo escolar. Porto Alegre: Artmed; 2002.

8. Oliveira ERA, Fiorin BH, Lopes LJ, Gomes MJ, Coelho $\mathrm{SO}$, Morra JS. Interdisciplinaridade, trabalho em equipe e multiprofissionalismo: concepções dos acadêmicos de enfermagem. Revista Brasileira de Pesquisa em Saúde 2011;13(4):28-34.
9. Saupe R, Cutolo LRA, Wendhausen ALP, Benito GAV. Competência dos profissionais da saúde para o trabalho interdisciplinar. Interface. 2005;9(18):521-536.

10. Souza DRP, Souza MBB. Interdisciplinaridade: identificando concepções e limites para a sua prática em um serviço de saúde. Rev. Eletr. Enf. 2009; 11(1):117-23.

11. Backes DS, Colomé JS, Erdmann RH, Lunardi VL. Grupo focal como técnica de coleta e análise de dados em pesquisa qualitativas. O Mundo da Saúde, São Paulo: Centro Universitário de São Camilo. 2011; 35(4):438-442.

12. Iervolino AS, Pelicioni MCF. A Utilização Do Grupo Focal Como Metodologia Qualitativa Na Promoção Da Saúde. Revista da Escola de Enfermagem da USP. 2001;35(2):115121.

13. Lefèvre F, Lefèvre AMC. O discurso do sujeito coletivo: um enfoque em pesquisa qualitativa (desdobramentos). 2 ed. Caxias do Sul: Educs; 2005. 256p.

14. Haddad AE, Pierantoni CR, Ristoff D, Xavier IM, Giolo J, Silva LB, (Org.). A trajetória dos cursos de graduação na área da saúde: 1991-2004. Brasília: Instituto Nacional de Estudos e Pesquisas Educacionais Anísio Teixeira; 2006.

15. Nogueira MI, Guedes CR. Da graduação biomédica à medicina de Família: aprendendo a se tornar um "médico da pessoa". Physis Revista de Saúde Coletiva. 2013; 23(2):439-460.

16. Costa JRB, Romano VF, Costa RR, Vitorino RR, Alves LA, Gomes AP, Siqueira-Batista R. Formação Médica na Estratégia de Saúde da Família: Percepções Discentes. Revista Brasileira de Educação Médica. 2012; 36(3):387-400.

17. Política Nacional de Extensão Universitária. Fórum de Pró-Reitores de Extensão das Universidades Públicas Brasileiras - FORPROEX. Plano Nacional de Extensão Universitária. Ilhéus: Editus; 2001.

18. Silva AFL, Ribeiro CDM, Silva Júnior AG. Pensando extensão universitária como campo de formação em saúde: uma experiência na Universidade Federal Fluminense, Brasil*. Interface (Botucatu).2013; 17(45):371-84.

19. Machado LM, Colomé JS, Beck CLC. Estratégia de Saúde da Família e o sistema de referência e de contra-referência: um desafio a ser enfrentado. Revista de Enfermagem da UFSM. 2011:31-40.

20. Higal EFR, Yamadai DM, Pelosoi GFB, Peresi CRFB, Panacionnei SZE, Mesquitai CMB. Estratégias para o Avanço da Integralidade na Visão de Professores e Estudantes. Revista Brasileira de Educação Médica. 2012; 36(4):470-477.

21. Garcia MAA, Pinto ATBCS, Odoni APC, Longhi BS, Machado LI, Linek MS, Costa NA. Interdisciplinaridade e integralidade no ensino em saúde. Rev. Ciênc. Méd. Campinas. $2006 ; 15(6): 473-485$. 
22. Aguilar-da-Silva RH, Scapin LT, Batista NA. Avaliação da formação interprofissional no ensino superior em saúde: aspectos da colaboração e do trabalho em equipe. Avaliação, Campinas. 2011; 16(1):167-184.

23. Mcnair R. The case for education health care students in professionalism as the core content of interprofessional education. Med Educ 2005; 39(5):456-464.

24. Fazenda I. Interdisciplinaridade - um projeto em parceria. São Paulo: Loyola; 1995.

25. Freire P. Pedagogia do Oprimido. 5.ed. Rio de Janeiro: Paz e Terra; 1978.

\section{CONTRIBUIÇÃO DOS AUTORES}

Renata Newman Leite Cardoso dos Santos - Desenho do estudo, coleta de dados, redação do texto, análise e interpretação dos dados, revisão final. Kátia Suely Queiroz Silva Ribeiro Desenho do estudo, interpretação dos dados e revisão da versão final do artigo. Ulisses Umbelino dos Anjos - Desenho do estudo, interpretação dos dados e revisão da versão final do artigo. Danyelle Nóbrega de Farias - Coleta de dados e revisão bibliográfica. Eleazar Marinho de Freitas Lucena - Análise dos dados e revisão do texto.

\section{CONFLITO DE INTERESSES}

Os autores declaram não haver conflito de interesses.

\section{ENDEREÇO PARA CORRESPONDÊNCIA}

Renata Newman Leite Cardoso dos Santos

Rua Dalva Filgueiras Athaíde, 30 - apto 703

Manaíra - João Pessoa

CEP 58038-512 - PB

E-mail: renatanewman@hotmail.com 